\title{
Obesity and lymphedema in geriatrics: combined therapeutical approaches
}

\author{
C Campisi", S Accogli, C Campisi, F Boccardo, C Campisi \\ From de Senectute: Age and Health Forum \\ Catanzaro, Italy. 5-7 December 2009
}

\section{Background}

Obesity combined with lymphedema, especially in geriatrics, is more than the sum of the two diseases [1] because it causes the diaphragm to be above its normal position, impairing its movement. As a consequence, a fundamental mechanism that supports lymph flow is significantly decreased. Obesity associated with lymphedema represents a cause of a highly invalidating condition. The purpose of this preliminary report is to propose a system of treatment, assessing the efficacy of a combined approach of lymphostatic disease in obese geriatric patients.

\section{Materials and methods}

A group of 6 geriatric patients affected by obesity (BMI>35) and lower limb lymphedema (II to III stage) had been enrolled in this preliminary study. Patients underwent a protocol of treatment of lymphedema by Complete Decongestive Therapy (CDT) according to Földi's method. Patients had been under observation for six-twelve months. They were evaluated during this period of follow-up at 1-3-6 and 12 months. After 12 months those patients who were not significantly responsive to non-operative methods, without any important regression of previous lymphedema staging, had a lymphangioscintigraphy performed in order to evaluate a right indication to lymphatic-venous microsurgical anastomoses [2].

impairment of lymph transport capacity index of lower limbs, underwent Derivative Lymphatic-Venous Bypass microsurgical procedure (LVA), performing a simultaneous operation at both groin sites (Figure1 and 2). Clinical and lymphoscintigraphic post-operative followup (evaluated at 3-5 years after surgery) showed a longlasting improvement, with a marked edema and volumetric reduction maintained by a proper lifestyle, remedial exercise, elastic stockings and periodical CDT procedures.

\section{Conclusions}

Considering the high incidence of obesity and the notunfrequent association with lower limb lymphedema, the method proposed of a combined approach in geriatric patients can represent an effective and long-lasting therapeutic solution.

Published: 19 May 2010

References

1. Földi M, Földi E: Földi's Textbook of Lymphology. Mosby-Elsevier, 2nd 2006.

2. Campisi C, Davini D, Bellini C, Taddei G, Villa G, Fulcheri E, et al: Lymphatic microsurgery for the treatment of lymphedema. Microsurgery. 2006, 26(1):65-9.

doi:10.1186/1471-2318-10-S1-A49

Cite this article as: Campisi et al:: Obesity and lymphedema in geriatrics: combined therapeutical approaches. BMC Geriatrics 2010 10(Suppl 1):A49.

\section{Results}

Two patients showed a significant reduction $(>50 \%$ in comparison to previous volumetry) of lower limbs affected by lymphstasis after CDT procedures, with a high level of patient satisfaction. Four patients, on the guide of lymphangioscintigraphy showing a relevant 


\section{Before}
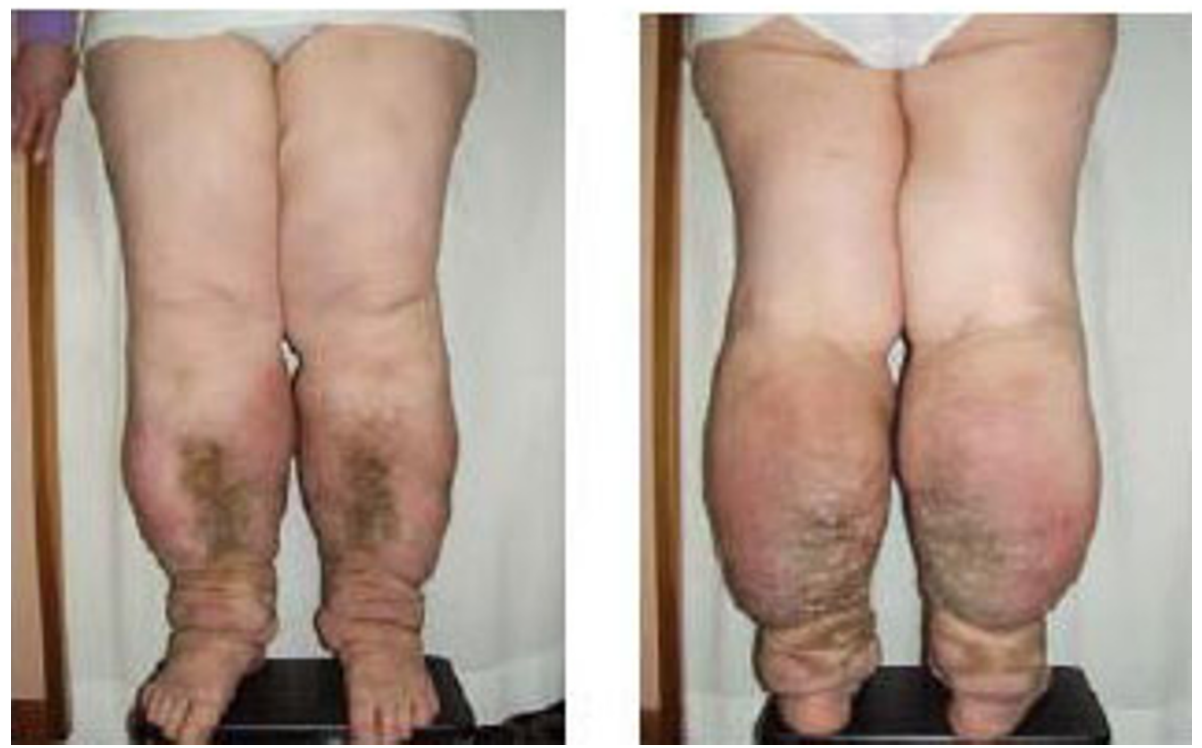

Figure 1 Obesity and lymphedema: an example of successful outcome of the combined therapeutical non-operative and microsurgical approaches.

\section{After}
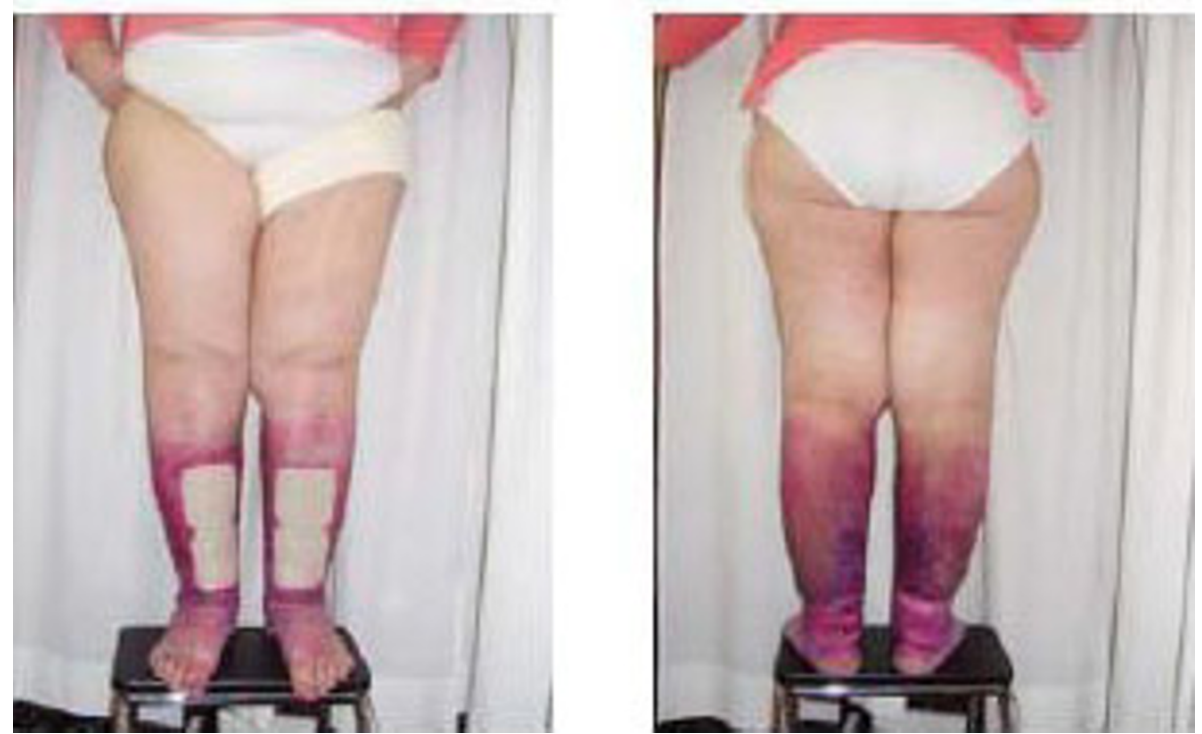

Figure 2 Obesity and lymphedema: an example of successful outcome of the combined therapeutical non-operative and microsurgical approaches, after. 